\title{
USING FINITE ELEMENT ANALYSIS IN THE 3D PRINTING OF METALS
}

\author{
Hussein ALZYOD*1 AND PÉTER FICZERE ${ }^{1}$ \\ ${ }^{1}$ Department of Railway Vehicles and Vehicle System Analysis, Budapest University of Technology and \\ Economics, Müegyetem rkp. 3, Budapest, 1111, HUNGARY
}

\begin{abstract}
Over the last few years, Additive Manufacturing, or as it is sometimes known, 3D printing, has become a significant research field for researchers worldwide. The necessity to increase the strength of materials and minimize their weight in the automotive and aviation industries has urged engineers and scientists to conduct more investigations and identify manufacturing methods to replace the classical ones. Additive manufacturing involves building a geometry layer by layer from a wide range of materials, which helps to utilize materials efficiently while minimizing the amount of waste produced as well as build complex, large geometries and light-weight components. Furthermore, it minimizes fabrication and processing times. In this paper, three different alloys were printed (TiAl6V4, AISi10Mg and 316L) using MSC Simufact software to investigate the effect of changing machines on the effective stress and surface deviation. Furthermore, thermal analysis as well as mechanical, thermal and thermomechanical calibrations were carried out to determine a parameter set consisting of the laser power, inherent strains, fraction of exposure energy and volumetric expansion factor.
\end{abstract}

Keywords: additive manufacturing, 3D printing, simufact additive.

\section{Introduction}

Over the last three decades, research into printing technology/additive manufacturing (AM) has progressed from fast prototyping to Industry $4.0[1,2]$. The Third Industrial Revolution took place in 1987 with the commercial exploitation of the first stereolithography (SLA) machine, which was developed by 3D systems and named SLA-1. After many editions of SLA machines in the early 90s, new AM principle technologies were launched, namely solid ground curing, fused deposition modeling and laminated object manufacturing [3,4].

This development was followed by many years of continuous improvement in AM technology, from resin to metal powders and from non-functional molding applications to the fabrication of medical implants [5]. AM methods have been characterized in the literature based on a variety of parameters, including direct or indirect process technology, the state of the raw materials, and the materials used. The state of the raw materials is the most commonly used as illustrated in Fig. 1.

$\mathrm{AM}$ is a revolutionary manufacturing technique proliferating as major industries transition from conventional to advanced production methods. Threedimensional printing is used in AM to convert engineering design files into fully functional and durable structures. After the components in the first layer have been bonded together using glue or heat, the second layer

\footnotetext{
*Correspondence: hussein.alzyod@edu.bme.hu
}

is constructed and the bonding procedure repeated, enabling the construction of previously unimaginable geometries. AM creates samples of products quickly, which is crucial since it reduces the traditional trial-and-error process, allowing novel ideas to enter the market more quickly. It may also be used to rapidly create bespoke metal items to replace old or broken industrial parts.

\subsection{Part-building technology}

Metal AM can be classified into four main categories as shown in Fig. 2: directed energy deposition, powder bed fusion, sheet lamination and binder jetting. Table 1 shows the thickness of layers in each process [6].

\subsection{Fields of application}

Metal 3D printing is the fastest-growing sector. Metal $\mathrm{AM}$ is increasingly being utilized to create final products

Table 1: The thickness of layers in the metal AM process [6].

\begin{tabular}{ll}
\hline Processing & $\begin{array}{l}\text { The thickness of } \\
\text { layers }(\mathrm{mm})\end{array}$ \\
\hline Directed energy deposition & $0.089-0.203$ \\
Powder bed fusion & $0.08-0.15$ \\
Sheet lamination & $0.1-0.19$ \\
Binder jetting & $0.089-0.203$ \\
\hline
\end{tabular}




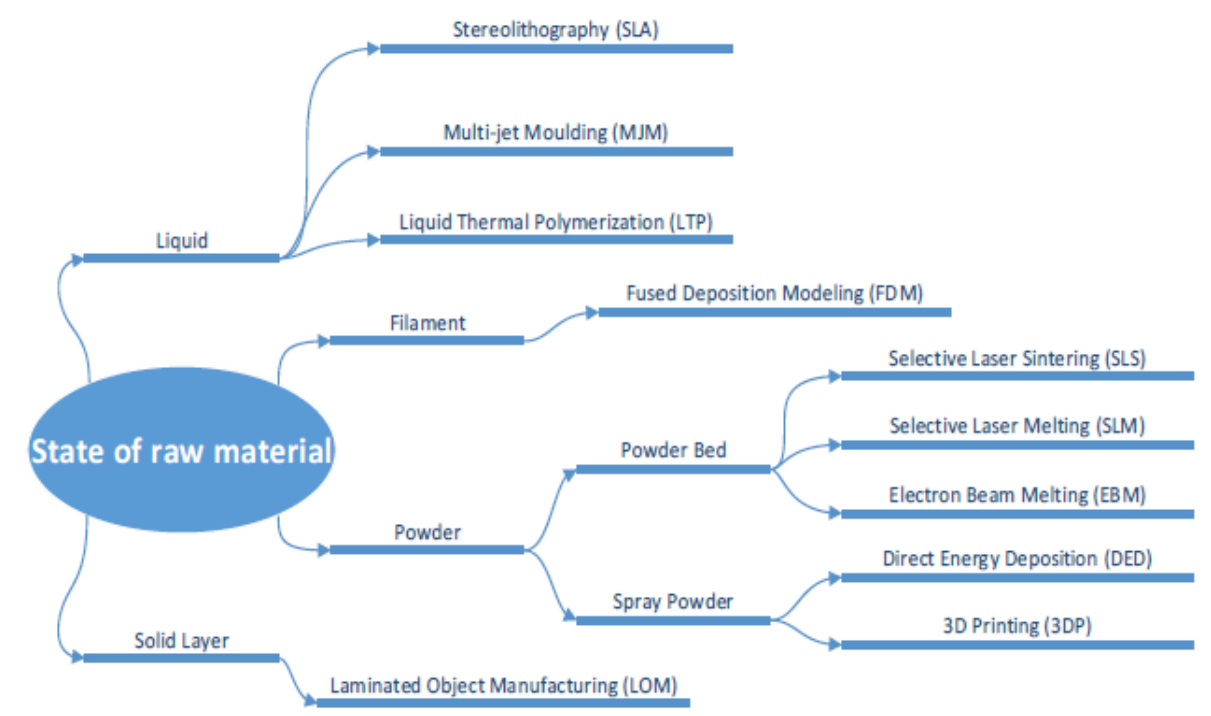

Figure 1: Types of Additive Manufacturing processes based on the state of raw materials [1].

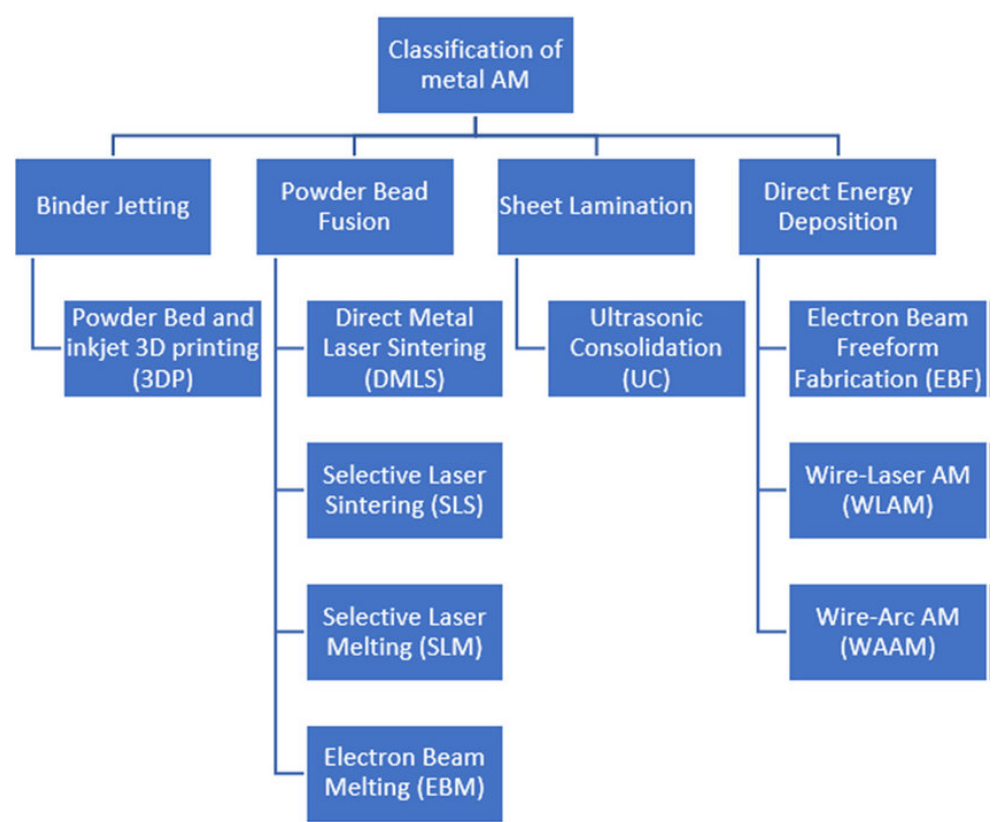

Figure 2: Classification of metal additive manufacturing technologies [5].

in many sectors like in the aviation, automotive, medical implants, material handling robotics as well as lifestyle and sport industries.

The aviation industry Companies in this sector are among the most well-known early investors of AM technology since they were among the first to upgrade from modest research projects to large-scale production runs. Norsk Titanium, Airbus, Alcoa and GE amongst others have begun serial production or are planning to invest in significant manufacturing operations within AM [7].

The automotive industry For many years, the automotive industry has utilized 3D printing to rapidly prototype single test components or entire visualization models.
Leading companies are experimenting with AM. Small series or customized production may be the first sectors to adopt this technology [7].

\subsection{Modeling approach in Simufact}

Many commercial companies, e.g., Autodesk, General Electric (via Geonx), MSC Software, ANSYS, etc., provide AM simulation, which enables designers to understand where the critical zones in a particular geometry are located and produce approximate first findings within a few hours. With this information, designers can iteratively develop their concept by taking all aspects into consideration using simulation software rather than wasting time and money on the actual manufacture of a possibly unsuitable design. Simufact is a piece of software 


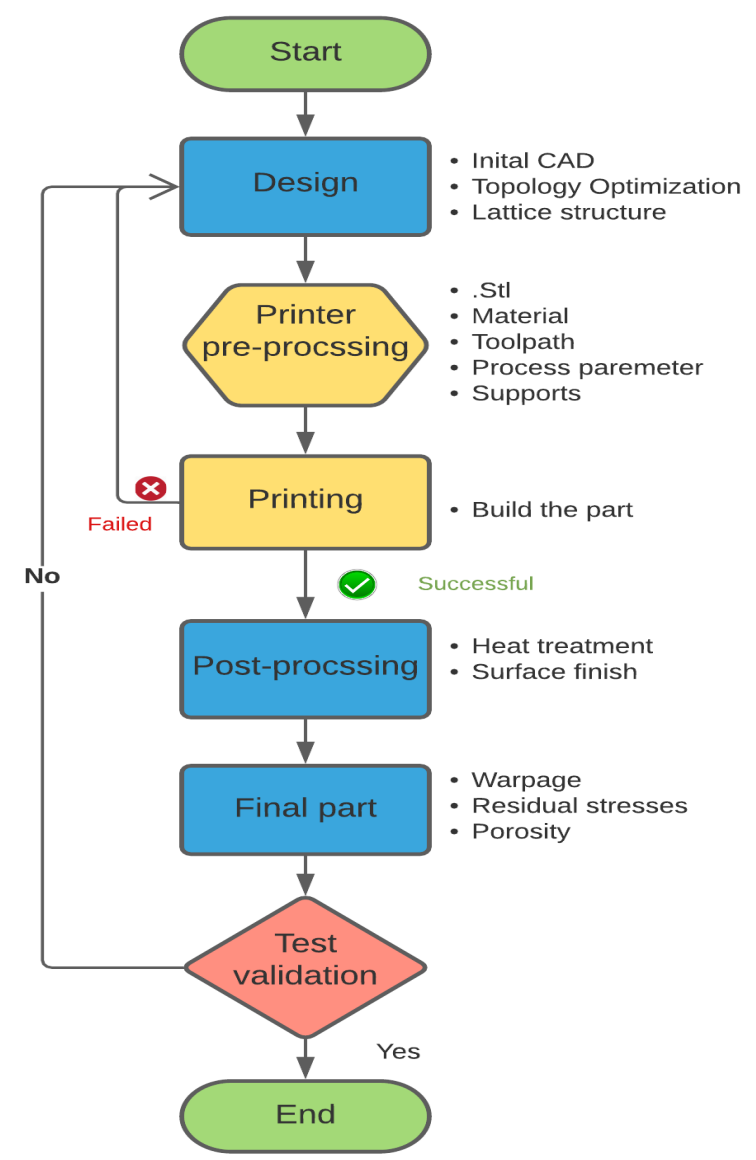

Figure 3: Additive Manufacturing workflow [own edition].

that uses finite element analysis to simulate metal AM by focusing on powder bed fusion processes. The difficulties with metal powder bed fusion AM can be addressed and analyzed by simulations [8].

As is shown in Fig. 3, the AM workflow to date has been one of the best traditional manufacturing technologies. The revision of AM goes through those processes to select the best predicted design parameters, build orientation, removal cutting, supporting structure, etc. [9].

Three-dimensional objects are constructed from several thin layers, each of which with a pattern defined by a Computer-aided design (CAD) that is exposed using a scanning laser for stereolithography or a pattern in the absence of a mask. Manufacturing information is obtained from $\mathrm{CAD}$ or other design software before being sliced into individual layers using the Standard Tessellation Language [10].

Sometimes, the building fails due to many reasons, e.g. supporting structures or a high degree of distortion. Once the best predicted design parameters have been chosen, construction commences and an inspection is made. If any defects are observed, the component is subjected to a process of trial and error to achieve the desired part. Alternatively, as illustrated in Fig. 4, a virtual model can be built to minimize the number of required physical builds

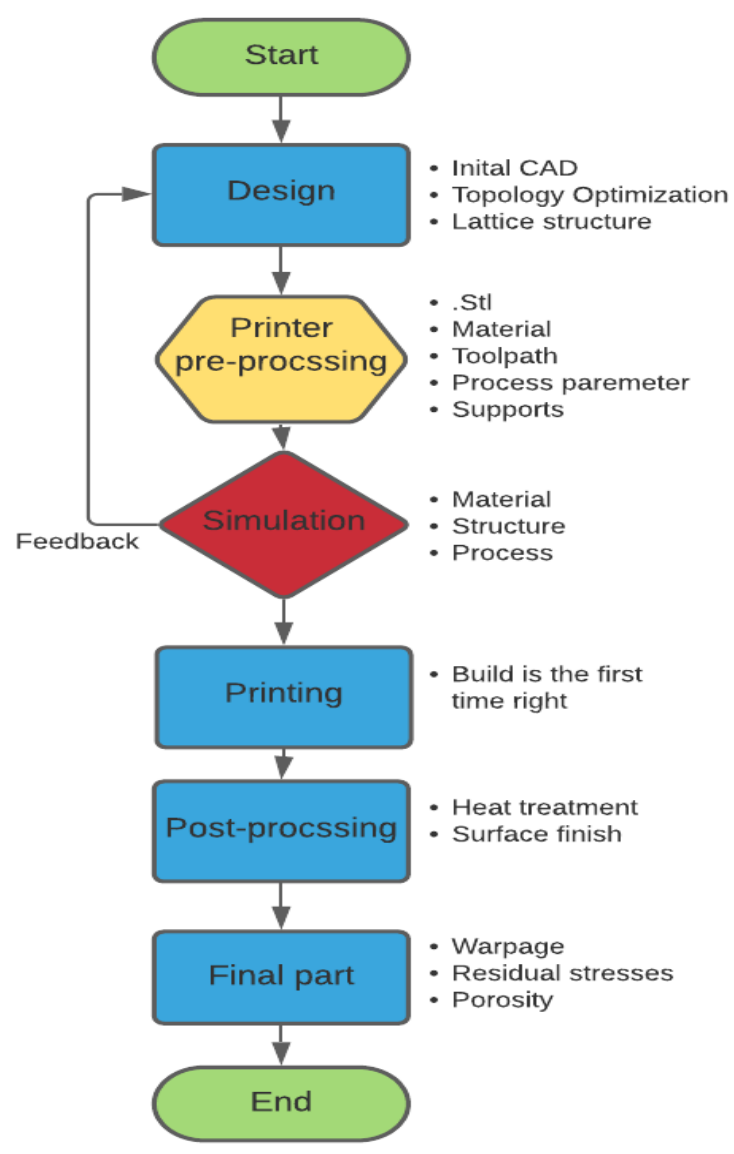

Figure 4: AM simulation workflow [own edition].

in the AM simulation workflow. In this virtual model, different approaches can be applied, e.g. various parameters, changing supporting structures, building orientation, and keeping treatment cutting and support removal to achieve the desired product free from any adverse effects of the process the first time it is constructed. Since a feedback loop is included in the simulation, the results of the process simulation can impact topology optimization. The significant benefits of this are that the formation of some of these parts may cost thousands of dollars and the trialand-error approach is quite undesirable.

\section{Design methodology and simulation}

Given that AM depends on many factors to determine the required parameter set to produce the desired part, e.g., laser power, fraction of exposure energy and volumetric expansion factor, experimental tests should be conducted to measure these parameters.

\subsection{Sample selection}

As is shown in Fig. 5 of the simulation, an aircraft component was chosen because of the complexity of its geometry and the importance of AM in the aerospace industry, 


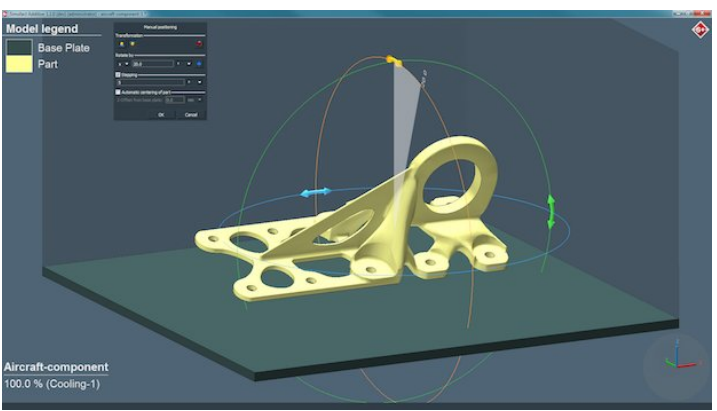

Figure 5: An aircraft component [9].

e.g., in increasing asset uptime, reducing costs, manufacturing lighter parts, enhancing durability and improving customer satisfaction.

\subsection{Material selection}

Three alloys (TiAl6V4, AlSi10Mg and 316L) were chosen with different properties to construct the sample for the purpose of investigating the effect of each material on the surface (shape) deviation with respect to the original sample.

\subsection{Machine selection}

Different types of 3D printing machines are available using this piece of software, e.g. two kinds of Electro Optical Systems (EOS) have been used, namely M280 and M400, the size and power of both differ.

\subsection{Thermal analysis}

The laser power has to be adjusted, moreover, the printing temperature has to be greater than the melting point of each alloy but less than its boiling point.

\subsection{Mechanical calibration}

The purely mechanical, macro-scale analysis approach of Simufact Additive requires the input of the so-called inherent strain values before the simulation. As is shown in Fig. 6, the inherent strain values are produced during the construction process as a result of plastic strain, thermal strain and phase transformations as shown in

$$
\epsilon^{\text {inherent }}=\epsilon^{\mathrm{th}}+\epsilon^{\mathrm{pl}}+\epsilon^{\mathrm{ph}} .
$$

Moreover, they depend on material properties and process parameters.

These strains can be calibrated from experiments, e.g. by measuring the degree of distortion after cutting a printed cantilever beam and running simulations to match the experimental distortion values that should be less than $3 \%$, or estimated based on the process. This paper used the empirical calibration by printing two cantilevers on the $X$ - and $Y$-axis as is shown in Fig. 7 that are $72 \mathrm{~mm}$

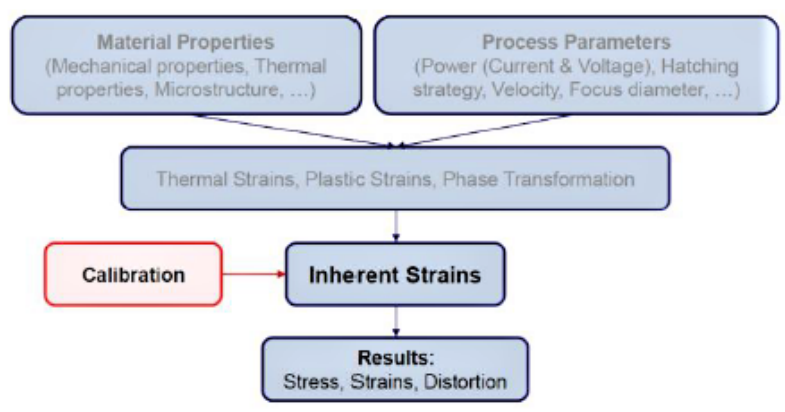

Figure 6: Inherent strain [9].

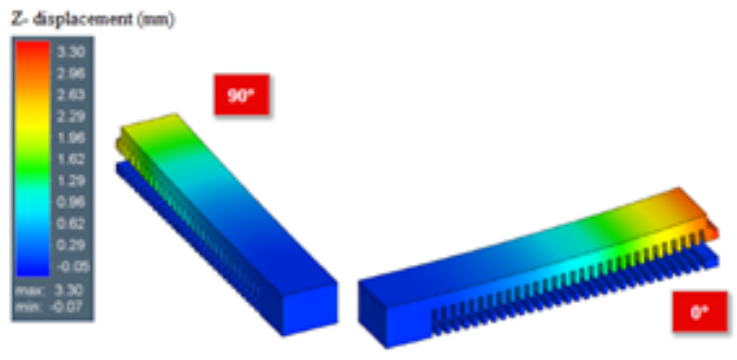

Figure 7: Printing two cantilevers [9].

in length, $12 \mathrm{~mm}$ in width, and $9 \mathrm{~mm}$ high. Afterwards, the cantilevers are cut in the middle of the teeth, so a particular part of them remains on the base plate. Finally, the maximum observed distortion in the $Z$-axis was measured as shown in Fig. 8.

Three values of strains are inputted into the build stage dialogue $\left(\epsilon_{x x}, \epsilon_{y y}, \epsilon_{z z}\right)$. These values are dimensionless and defined in three directions, $X, Y$, and $Z$. Default values will be shown in the dialogue [9]:

$$
\epsilon_{x x}=-008, \epsilon_{y y}=-0.003, \epsilon_{z z}=-0.030 .
$$

The mechanical calibration was carried out for the three alloys, namely TiAl6V4, AlSi10Mg and 316L, to calculate the inherent strains.

\subsection{Thermomechanical calibration}

The thermomechanical analysis requires that the fraction of the exposure energy and the volumetric expansion factor have been calculated before the simulation is started.
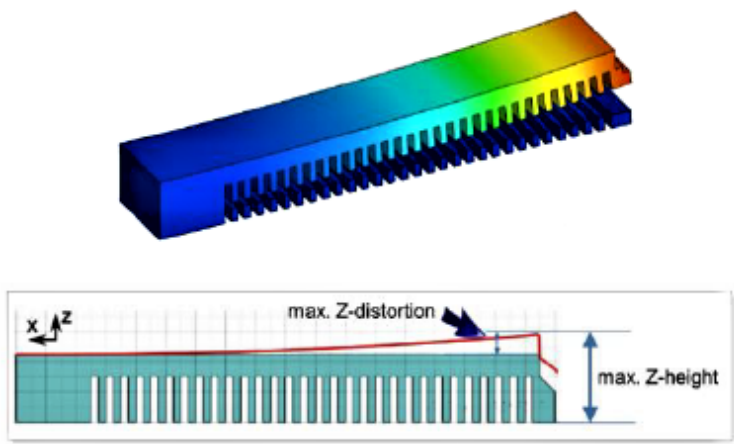

Figure 8: Measuring the distortion in the $Z$-axis [9]. 


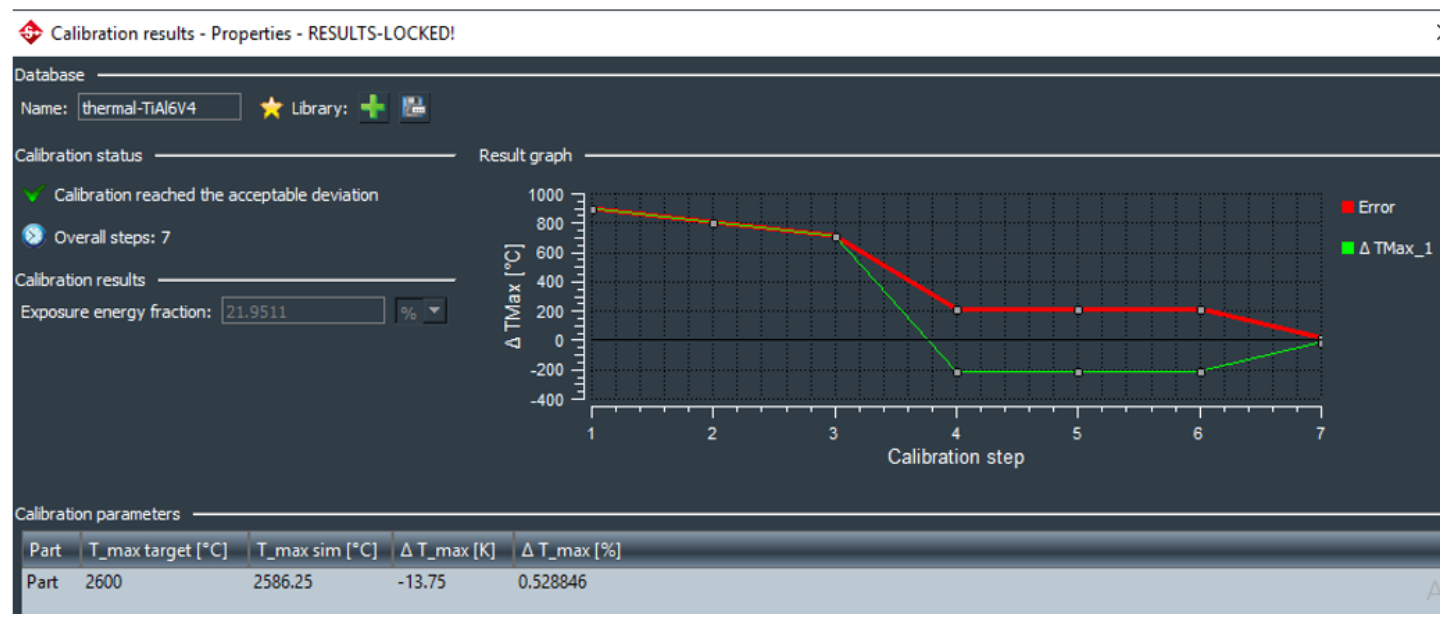

Figure 9: Thermal calibration

Since these values depend on material properties and machine parameters, they need to be carefully calibrated, which should be done experimentally.

\subsection{Fractions of exposure energy}

The fraction of exposure energy is a term that is responsible for the peak temperature of the process. During the exposure time, the energy predominantly (but not only) melts the powder. The rest of the powder reheats the solid material after the exposure time. For the purpose of thermomechanical calibration, it is recommended to carry out the thermal calibration first to determine the optimized exposure energy and then conduct the thermomechanical calibration with the optimized fraction of the exposure energy to calibrate the volumetric expansion factor.

The calibration was carried out using a specimen of a cantilever. The peak temperature has to be measured during the process to determine the maximum degree of distortion in the $Z$-axis after cutting, as is shown in Fig. 8. The measured point was chosen on the upper surface because the temperature is the highest there and the average will be recorded between the two surfaces. Thermal calibration of the three alloys, that is, TiAl6V4, AlSi10Mg and $316 \mathrm{~L}$, was carried out to determine the fraction of exposure energy. The thermal calibration of TiAl6V4 is shown in Fig. 9.

\subsection{Volumetric expansion factor}

The volumetric expansion factor accounts for the thermal expansion and shrinkage effects caused by the modelling approach, that is, by combining multiple powder layers and repeated heating of the heat source in one element layer. The volumetric expansion factor can either be set to isotropic in all directions or anisotropic for each individual order and scales the thermal expansion factor of the material. Volumetric expansion is the change in the volume of a given mass with temperature. The thermal expansion factor is a measure of the change in volume in three dimensions as is shown in Eq. ??:

$$
V=3 \alpha V_{\mathrm{o}} \Delta T
$$

This equation is usually written as:

$$
V=\beta V_{\mathrm{o}} \Delta T
$$

where $\beta=3 \alpha$ denotes the volumetric expansion factor, $V_{\mathrm{o}}$ represents the original volume and $\Delta T$ stands for the change in temperature.

\section{Results}

Different machines and materials were used to print an aircraft component. As illustrated in Table 2, changing the machine does not have any effect on the design or features of the sample. The effective stress in MSC Simufact was implemented once the printed aircraft component had been compared to calculations regarding residual stresses using Stoney's equation. The effective stress of both the M280 and M400 machines is identical. For the alloys TiAl6V4, AlSi10Mg and 316L, the stresses were $1260 \mathrm{MPa}, 370 \mathrm{MPa}$ and $680 \mathrm{MPa}$, respectively. When the M280 machine was used, the surface deviation, representing the difference between the designed and printed samples, of all three alloys did not change. However, when the M400 machine was used, the surface deviation in each alloy varied.

The laser power of the machine, which is responsible for fusing the powder, is one of the most crucial elements to modify. As a result, the printing temperature of a material, which is roughly twice as high as its melting point, must be higher than its melting point but lower than its boiling point. For each alloy, the changes in laser power as well as the measured temperature are shown in Table 3. For TiAl6V4, $200 \mathrm{~W}$ is a suitable power, while the optimal laser power for AlSi10Mg and 316L is $100 \mathrm{~W}$.

The mechanical calibration was carried out to identify the inherent stresses. For measuring the distortion in 
Table 2: The effects of the machines and alloys.

\begin{tabular}{|c|c|c|c|c|}
\hline \multirow{3}{*}{$\begin{array}{l}\text { Material } \\
\text { Used }\end{array}$} & \multirow{3}{*}{$\begin{array}{l}\text { EOS } \\
\text { Machine }\end{array}$} & \multirow{3}{*}{$\begin{array}{l}\text { Effective } \\
\text { Stress [MPa] }\end{array}$} & \multirow{2}{*}{\multicolumn{2}{|c|}{$\begin{array}{l}\text { Surface } \\
\text { Deviation }[\mathrm{mm}]\end{array}$}} \\
\hline & & & & \\
\hline & & & Max & Min \\
\hline \multirow[t]{2}{*}{ TiAl6V4 } & M280 & 1262.03 & 0.17 & -0.16 \\
\hline & M400 & 1288.13 & 0.88 & -0.93 \\
\hline \multirow[t]{2}{*}{$\mathrm{AlSi} 10 \mathrm{Mg}$} & M280 & 370.01 & 0.13 & -0.12 \\
\hline & M400 & 370.02 & 0.36 & 0.26 \\
\hline \multirow[t]{2}{*}{$316 \mathrm{~L}$} & M280 & 683.19 & 0.17 & -0.16 \\
\hline & M400 & 680.65 & 0.16 & -0.15 \\
\hline
\end{tabular}

Table 3: Adjusting the laser power.

\begin{tabular}{llll}
\hline Alloy used & $\begin{array}{l}\text { Melting } \\
\text { point }\left(\mathrm{C}^{\circ}\right)\end{array}$ & $\begin{array}{l}\text { Laser } \\
\text { power }(\mathrm{W})\end{array}$ & $\begin{array}{l}\text { Printing } \\
\text { temperature }\left(\mathrm{C}^{\circ}\right)\end{array}$ \\
\hline TiA16V4 & 1600 & 200 & 2650 \\
AlSi10Mg & 660 & 200 & 4220 \\
& & 150 & 3098 \\
& & 100 & 2084 \\
316L & \multirow{2}{*}{1400} & 200 & 4454 \\
& & 150 & 3500 \\
& & 100 & 2463 \\
\hline
\end{tabular}

the beam, two points on the cantilever were chosen at heights of 3.09 and $2.01 \mathrm{~mm}$. The simulation repeatedly printed the specimen until the permissible distortion error became less than $3 \%$. The sample was printed seven times to achieve an acceptable degree of distortion. Table 4 shows the inherent strains of the three alloys.

Since the volumetric expansion factor is a crucial parameter in $3 \mathrm{D}$ printing, the simulation carried out the thermomechanical calibration to determine the volumetric expansion factor, as is shown in Table 5.

\section{Conclusion}

The simulation tool Simufact was used to print a component of an airplane out of different alloys using AM technology to determine the specified parameter and carry out virtual printing. As indicated in Table 3, since the laser power has a massive effect on the printing temperature, it should be regulated carefully. Furthermore, as indicated in Tables 4 and 5, in order to determine the inherent strains and volumetric expansion factor, the component must be printed multiple times.

\section{REFERENCES}

[1] Hernandez Korner, M.E.; Lambán, M.P.; Albajez, J.A.; Santolaria, J.; Ng Corrales, L.d.C.; Royo, J.: Systematic literature review: integration of additive manufacturing and industry 4.0. Metals, 2020. 10(8),1061 DOI: 10.3390/met10081061

[2] Alex, S.; Árpád, T.: XBRL utilization as an automated industry analysis. Hung. J. Ind. Chem., 2020, 48(1), 131-138 DOI: 10.33927/hjic-2020-19
Table 4: Inherent strains of TiAl6V4, AlSi10Mg and 316L.

\begin{tabular}{lllll}
\hline Metal & $\epsilon_{x x}$ & $\epsilon_{y y}$ & $\epsilon_{z z}$ & $N_{\mathrm{s}}$ \\
TiAl6V4 & -0.00488099 & -0.0022865 & -0.3 & 7 \\
AlSi10Mg & -0.00637062 & -0.0016680 & -0.3 & 18 \\
316L & -0.0198353 & -0.0025170 & -0.3 & 3 \\
\hline
\end{tabular}

$N_{\mathrm{s}}$ : Number of samples printed

Table 5: Volumetric expansion factors for TiAl6V4, AlSi10Mg and 316L.

\begin{tabular}{lll}
\hline Metal & $\begin{array}{l}\text { Volumetric expansion } \\
\text { factor }\end{array}$ & $\begin{array}{l}\text { Number } \\
\text { of steps }\end{array}$ \\
\hline TiAl6V4 & 0.821869 & 18 \\
A1Si10Mg & 0.60999 & 2 \\
316L & 0.307238 & 24 \\
\hline
\end{tabular}

[3] Attaran, M.: The rise of 3-D printing: The advantages of additive manufacturing over traditional manufacturing. Bus. Horiz., 2017, 60(5), 677-688 DOI: 10.1016/j.bushor.2017.05.011

[4] Gábora, A.; Varga, T. A.; Kozma, I.; Beke, S.; Mankovits, T.: 3D Geometrical Reconstruction of Closed-Cell Aluminum Foam Using CT Images. in InCell 2019: Book of Abstracts of the International Conference on Multifunctional Cellular Materials (UA Editora Universidade de Aveiro.), page 74 ISBN: 978-972-789-611-0 https://ria.ua.pt

[5] Li, J.Z.; Alkahari, M.R.; Rosli, N.A.B.; Hasan, R.; Sudin, M.N.; Ramli, F.R.: Review of wire arc additive manufacturing for $3 \mathrm{D}$ metal printing, Int. J. Autom. Technol., 2019, 13(3), 346-353 DOI: 10.20965/ijat.2019.p0346

[6] Loughborough, U.: the 7 Categories of Additive Manufacturing, 2019, https://www.lboro.ac.uk

[7] Software solutions, S.S.S.A.S.: Additive Manufacturing Technology, 2017, https://www.simufact.com

[8] Mankovits, T.: Numerical analysis of unit cell models for orthopedic applications, IOP Conf. Ser: Mater. Sci. Eng., 393, 012019 DOI: 10.1088/1757899x/393/1/012019

[9] Software solutions, S.S.S.A.S.: English webinar on the entire portfolio of MSC Software for Additive Manufacturing, 2017, https://www.simufact.com

[10] Rasiya, G.; Shukla, A.; Saran, K.: Additive Manufacturing-A Review, Mater. Today: Proc., 2021, 47(1), 6896-6901 DOI: 10.1016/j.matpr.2021.05.181

[11] Schelhorn, L.; Gosch, M.; Debeugny, L.; Schroter, P.; Schwarz, W.; Soller, S.: Optimal Design and Process Simulation for Additive Manufacturing, in 8th European Conference for Aeronautics and Space Sciences, EUCASS2019-FP0354, Madrid, Spain, 1-15 DOI: 10.13009/EUCASS2019-354 